\title{
UN MANUAL PARA LA FORMACIÓN DE LAS COMADRONAS ESPAÑOLAS (1929)
}

\section{Francisco Herrera Rodríguez (*)}

(*) Profesor titular de Historia de la Enfermería.

Escuela Universitaria de Ciencias de la Salud. Universidad de Cádiz.

Correspondencia:

Francisco Herrera Rodríguez.

C/ Santo Domingo de la Calzada, n 11, 31A. 11012 Cádiz

\section{RESUMEN}

ELEMENTOS GEL ARTE DE RAREAR,

ccuressyos

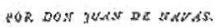

PARTE PRLBERA

\section{MANUAL FOR EDUCATION OF SPANISH MIDWIVES.}

\section{SUMMARY.}

The present study examines Piskacek's Manual for Midwives, published in Spain, 1929, a crucial year for the associative movement of the aforementioned professionals.

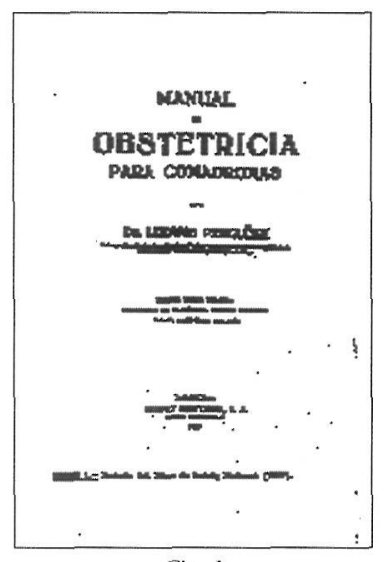

Fig. I

The book "Manual para Matronas", written by an obstetrician with 30 years of experience in the education of midwives is analysed in its essential directional lines with some particular considerations for the situation midwives found themselves in during this period in the history of our country. The Manual underlines the lack of texts written in those years - the 1920's - specifically as regarding the education of midwives, who often made their own study using medical doctors' and obstreticians' compendia. It also underlines the importance of foreign influence in the education of Spanish sanitary staff. 


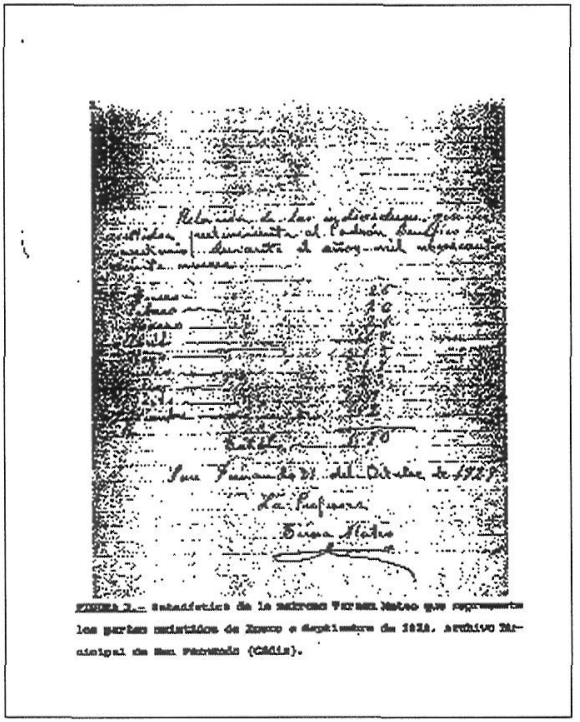

Fig. 2

"Después de cortarle el ombligo la comadrona se puso a quitarle con un trapo el unghento azul que le cubría el cuerpo, alumbrada por Aureliano con una lámpara. Sólo cuando lo voltearon boca abajo se dieron cuenta de que tenía algo más que el resto de los hombres, y se inclinaron para examinarlo. Era una cola de cerdo".

García Márquez,

"Cien años de soledad"

"Mi llegada a este mundo fue coreada por los gritos y aplausos de una mona babuina mientras mi madre, a tientas en la oscuridad, se partía el cordón con los dientes. Ella tuvo que esperar a que amaneciera para verme el rostro..."

Manuel Vicent,

"Balada de Caín"
El objetivo de este artículo es analizar el Manual de Obstetricia para comadronas del tocólogo austriaco Ludwig Piskacek, a la luz de la situación en España de estas profesionales en torno al año 1929 , fecha de publicación del referido libro (véase figura 1). Dada la experiencia de este médico en la formación de las comadronas vienesas, pensamos que tiene interés vaciar el contenido de su Manual de cara a entender las funciones y tareas que específicamente realizaban las comadronas en la época señalada. Obviamente, este libro de Piskacek no fue el único disponible en el mercado para la preparación de las comadronas españolas. Sin duda, algunas de estas mujeres aún dispondrían del clásico Manual del Arte de Obstetricia, para uso de las matronas, publicado en Madrid en el año 1866, por Francisco Alonso Rubio; autor que debemos situar en la tradición publicística para la formación de estas profesionales junto, por ejemplo, a Damià Carbó, que en 1541 sacó a la luz el conocido Libro del arte de las comadres... (Martínez Molina, 1994) y a Juan de Navas que en 1795 ofreció a la imprenta los Elementos del arte de partear (Herrera y Doña, 1989).

Pero precisando más aún podemos indicar que en los años veinte de la actual centuria se recomendaron en España a las matronas otros libros, entre los que podemos destacar los siguientes: el Manual de obstetricia para comadronas de Torre Blanco, tocólogo de la Casa de Maternidad de Madrid; El alumbramiento normal y su patología (1927), de Manuel de Usandizaga (Herrera, 1995); La práctica obstétrica y ginecológica en la medicina rural (1930), de Aza y Carreras. También se recomendaron los Tratados de Obstetricia o de Ginecología de autores extranjeros como Bumm,

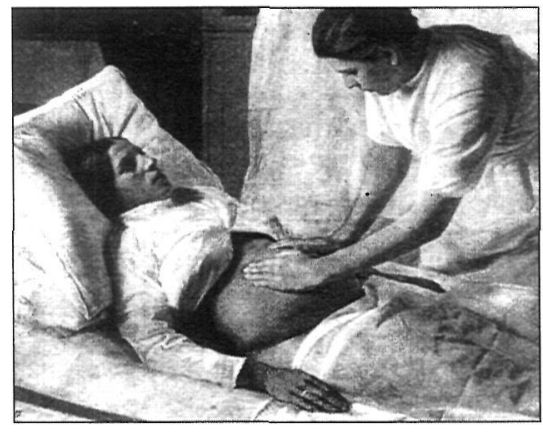


Fabre y Stoeckel. Como se puede comprobar no era muy abundante entre nosotros el material bibliográfico dedicado específica y exclusivamente a la formación de comadronas.

Antes de comentar el libro de Piskacek hay que precisar algunas cuestiones. Por ejemplo, debemos plantearnos por qué en 1929 la editorial Salvat decide traducir del alemán un libro para comadronas. La respuesta, evidentemente, es que había demanda de este tipo de libros especializados. Hay que tener en cuenta que en la Gaceta del 18 de septiembre de 1926 y del 31 de agosto de 1928 se reformó el plan de estudios de las comadronas. La primera de las normativas citadas incluye que para cursar esta carrera se debe estar en posesión del título de Bachiller elemental y la segunda normativa señala que deben cursarse dos años de enseñanza teórico-práctica en las "Escuelas Matronales que se crearán al efecto", debiendo el director de la institución acreditar los estudios de la alumna y que ha asistido un mínimo de cien partos dentro de la Escuela; sólo entonces, la aspirante al título estará en condiciones de solicitar examen teórico-práctico en la Facultad de Medicina correspondiente (Martínez de Aguirre, 1929). Debemos precisar que la prensa profesional de las matronas denunció, por ejemplo, en Sevilla, que en esta población no se había creado la Escuela, de acuerdo con la mentada normativa de 1928 ("LA MATRONA HISPALENSE", 1930). Aún así sabemos que en el curso 192728 se matricularon en nuestro país un total de 1.380 aspirantes al título de matrona, bajando las matriculaciones en los años siguientes, produciéndose luego la recuperación durante la Segunda República (Siles, 1996). Sin duda, el elevado número de matriculadas durante el curso académico 1927-28 fue uno de los factores que alentó a la editorial Salvat a editar en castellano el libro que glosamos en el presente artículo. También hay que tener presente que los aspirantes al título de practicante en nuestro país debían preparar un temario muy amplio relacionado con la Obstetricia, lo que les convertía en potenciales compradores del libro. A todo esto hay que añadir que en 1929 las matronas españolas se muestran especialmente activas: reuniéndose en el mes de mayo en Madrid, en el Congreso Nacional de
Matronas, y en el mes de septiembre en Barcelona, en el contexto del Primer Congreso Internacional de Matronas (Lasarte, 1995). En estos foros reivindican que se tomen medidas legales contra el intrusismo, que se creen plazas de matronas en la Beneficencia y que se imponga la colegiación obligatoria. Precisamente, esta última reivindicación es solicitada en paralelo para cada uno de sus colectivos por el presidente de la Federación de Practicantes, Rafael Fernández Carril, y por la presidenta de la Federación de Matronas, Concepción Marín ("LA MATRONA HISPALENSE", 1929). Los practicantes conseguirán la colegiación oficial obligatoria en 1929 y las matronas poco después, concretamente, por una Real Orden de 7 de mayo de 1930 (De La Peña, 1943) (Herrera y Lasarte, 1996). Además, en 1929, algunos colegios de matronas organizaron para estas profesionales cursos de ampliación de estudios, con gran concurrencia, como el que impartió el doctor Jacinto Ovín Corte en el colegio sevillano ("LA MATRONA HISPALENSE", 1929).

En este contexto, pues, aparece el libro del tocólogo austriaco Piskacek. Un libro que se encuentra estructurado en cuatro partes. En la primera se plasma una descripción general muy breve del cuerpo humano y en la segunda se estudia la pelvis y los órganos genitales femeninos. A continuación, más extensamente, se desarrollan todos los pormenores del embarazo, parto, puerperio y los cuidados y la alimentación del recién nacido. Y, por último, encontramos el estudio de las enfermedades y anomalías de las embarazadas, parturientas, puérperas y de los recién nacidos y niños de pecho.

Precisamente, esta división neta entre el estudio de lo normal y lo anómalo no sólo busca una diferenciación estructural o pedagógica, sino que es también una forma de hacer énfasis el autor sobre la circunstancia de que la matrona debe detectar prontamente la "anomalía" y solicitar el auxilio del facultativo. Al hilo de este comentario, debemos aclarar que en nuestro país se publicó una Real Orden 11 de diciembre de 1928 determinando que la función de las matronas es la asistencia a los partos normales, pero como la condición fisiológica de 
éstos sólo puede ser determinada por los médicos, la intervención de estas profesionales estará siempre supeditada a la indicación previa del facultativo encargado de la asistencia (De La Peña, 1943). Esta normativa no fue bien recibida por algunas matronas que veían peligrar su autonomía profesional. En cambio, algunos médicos se mostraron satisfechos con la medida, compruébese esto en el párrafo que transcribimos a continuación:

"No se asusten nuestras matronas con esa disposición; nada les perjudica, pues su misión será siempre necesaria porque es útil y la ejercerán así con más tranquilidad..." (Ríos Sarmientos, 1930).

Efectivamente, a pesar de la referida normativa a la matrona española le quedaba aún un ámbito de trabajo suficientemente importante para su asentamiento profesional, pero lógicamente estas profesionales tratan de proteger sus intereses intentando fortalecerse ante otros colectivos como el de los médicos o el de los practicantes. La realidad es que en estos años que estudiamos la matrona juega un papel muy importante en la asistencia de los partos eutócicos. Esta afirmación que hacemos la hemos querido corroborar de una forma práctica a través de fuentes documentales concretas. En este sentido hemos tenido la suerte de localizar un expediente que compendia información de los años 1925 a 1929 sobre la asistencia a las parturientas que pertenecían al padrón benéfico-sanitario de la población gaditana de San Fernando (LEGAJO 1180: A.M.S.). Este expediente es interesante porque plasma la labor de las matronas en la localidad mencionada en los años referidos. El acopio de esta documentación parte de la necesidad que tiene en esas fechas el Ayuntamiento de San Fernando de conocer el número de partos normales y distócicos asistidos por los médicos y matronas de la localidad entre 1925 y 1929, para con esta información poder justificar la creación de nuevas plazas de sanitarios para la Beneficencia Municipal. De uno de estos documentos extraemos el siguiente párrafo que viene a corroborar lo que estamos diciendo:

"En esta población es muy general en todas las clases sociales y en especial en los pobres, requerir el auxilio para los partos a las matronas y sólo a los médicos en los casos que se presentan difíciles...".

Pues bien, a petición del Ayuntamiento los médicos y matronas ofrecen la información que se les demanda. Elegimos el escrito de Manuel Pece Casas, médico titular del cuarto distrito:

"...la carencia de datos que puedo aportar con respecto al particular que se interesa, pues ocurre que los partos normales son asistidos por las profesoras de la localidad y cuando se presenta un parto distócico, solicitan la intervención de los especializados que existen en la ciudad. Sin embargo, de una manera muy aproximada, puedo manisfestar a V.E. que durante el período de tiempo que se indica he asistido unos cuarenta partos distócicos, incluyendo como tal las extracciones placentarias".

Las dos matronas encargadas de asistir en San Fernando en los referidos años a las parturientas del padrón benéfico-sanitario son Isabel Arellano Pereira y Teresa Mateo Rubiales, aunque después de estudiada la situación el Ayuntamiento pretende aumentar el personal con una matrona más, con una dotación económica de 900 pesetas, mientras que la plaza de médico tocólogo estaba dotada con 3.000 pesetas. De esta documentación que comentamos destaca la intensa actividad de estas matronas de la Beneficencia Municipal, sino compruébese lo que decimos estudiando simplemente el número de partos asistidos entre 1925 y 1929 por Teresa Mateos:
-1925: 163 partos asistidos.
-1926: 138
-1927: 93
-1928: 114
-1929: 170
" (véase figura 2).

Una vez que hemos realizado estas matizaciones, analizando la dicotomía asistencial que plantea el tocólogo austriaco en su libro, a la luz de la realidad española, bueno será que volvamos nuevamente a su contenido y nos centremos sobre todo en lo que Piskacek plantea sobre la conducta de la matrona en las complicaciones del embarazo, pues- 
to que damos por sentado que este libro presenta apartados muy ilustrativos y pedagógicos sobre el interrogatorio obstétrico, la exploración, el instrumental y la explicación detallada de la evolución de un parto normal. No duda Piskacek en subrayar la necesidad de la presencia médica en los casos siguientes: cuando tarda la expulsión de la placenta; en los ataques de eclampsia; cuando la mujer tiene una pelvis estrecha; cuando se aprecia la situación anómala del útero grávido; en las presentaciones anormales del feto (vértice, cara, frente, nalgas, transversales y oblicuas) y en los casos de aborto y de sufrimiento fetal.

Debemos aclarar que todo el libro gira en torno a esquemas, dibujos y fotografías (véase figura 3) y epígrafes muy claramente redactados, en los que se precisa cual debe ser la conducta de la matrona ante cada caso. Leamos el siguiente párrafo que ilustra perfectamente lo que decimos:

"Al principio de cualquier hemorragia todos los esfuerzos de la comadrona deben ir encaminados a detener la hemorragia (...)Si de esta manera la hemorragia no se detiene, debe enviarse a buscar el médico".

Piskacek es consciente de que el "vulgo" piensa que las comadronas son también expertas en el reconocimiento y tratamiento de las mujeres con enfermedades ginecológicas, cosa que escapa enteramente de su labor profesional. Sin embargo, el tocólogo austriaco resalta que la matrona debe conocer bien el curso clínico del cáncer de útero y del cáncer de mama, ya que puede jugar un papel muy importante derivando al médico a las mujeres que le consulten por hemorragias irregulares, flujos vaginales o por un nódulo duro en la glándula mamaria.

Igualmente, en este libro se ilustra a las comadronas sobre la alimentación natural del recién nacido; los cuidados durante este proceso que deben dispensarse a las glándulas mamarias de la madre; los fundamentos prácticos del destete; la lactancia artificial del niño e incluso las condiciones que debe poseer una nodriza para ser recomendada a la familia por la comadrona; y también como se deben aplicar las técnicas de reanimación en caso de asfixia del recién nacido.

Este campo de acción de las matronas en torno a los cuidados de recién nacido, perfectamente subrayado por Piskacek, también fue reivindicado en nuestro país por las matronas e incluso algunos médicos reclamaron que esta función fuera desarrollada por estas profesionales. Léase, por ejemplo, la opinión del comandante médico Eduardo Delgado, que fue publicada en 1929, en las páginas de "La Matrona Hispalense":

"Ofrezcamos nuestro apoyo a la clase matronal española (...) ha de llegar hasta ella una amplia participación sanitaria, especialmente en cuanto a la puericultura se refiere, constituyendo el debido enlace entre la madre, el niño y el médico".

Finalmente, debemos precisar algunas cuestiones. Por ejemplo, que este libro vertido al castellano en 1929, fue editado por primera vez por Piskacek en 1895 y del prólogo de la primera edición podemos extraer una serie de datos complementarios: el propio tocólogo reconoce la influencia que ha tenido en la redacción de este libro de otros autores como Spath, Dohm, Credé-Leopold, Rubeska y Schutze; además de subrayar la participación del doctor Ricardo Klunzniger, autor de la mayor parte de las figuras y responsable por tanto de la riqueza iconográfica de este libro. Todos estos aspectos deben tenerse presente a la hora de valorar la influencia extranjera en la formación de los auxiliares sanitarios españoles.

En definitiva, en este artículo hemos querido resaltar la importancia de la aparición de este libro de Piskacek, dada las escasas referencias bibliográficas específicas que poseían las comadronas españolas para su formación, debiendo realizar sus estudios en muchas ocasiones en manuales y tratados de Obstetricia que estaban concebidos para la formación de médicos y no para la de estas profesionales. Por último, quisiéramos significar que la aparición en 1929 de este libro en el mercado coincide, como hemos precisado ya, con las múltiples actividades y reivindicaciones que en esas fechas realizan las matronas españolas. 
BIBLIOGRAFÍA.

Herrera, F.(1995) El manual de Enfermería de Manuel Usandizaga (1938). En: Qalat Chábir 3: 101-105.

Herrera, F. y Doña, F. (1989) La aportación de Juan de Navas a la formación de comadronas (1795). En: Minutos Menarini 164. 24- 27.

Herrera, F. y Lasarte, J.E. (1996) La prensa andaluza de los practicantes y matronas (1916-1939). En: I Congreso Nacional de Historia de la Enfermería. Libro de Ponencias y Comunicaciones. Fundación Uriach, Barcelona: 169-174.

Lasarte, J.E. (1995) La prensa de las profesiones auxiliares sanitarias en Andalucía. Estudio socio-profesional (1916-1939). Universidad de Cádiz, Barcelona. Edición en Microfichas.

Martínez Molina, A. (1994) Estudio histórico de la enfermería en España: La profesión de la matrona según el tratado de Damià Carbó (siglo XVI). Fundación de Enfermería Internacional, Sueca.

Peña, J. De La (1943) Legislación Sanitaria vigente de Medicina. Editorial: Tip. y Enc. de Senén Martín, Ávila.

Siles, J. (1996) Pasado, presente y futuro de la enfermería en España (Perspectiva histórica y epistemológica). Cecova. Fundación "José LLopis", Alicante.

\section{FUENTES}

Piskacek, L.(1929) Manual de Obstetricia para Comadronas. Salvat editores, S.A., Barcelona.

Ríos Sarmientos (1930) Una acertada disposición. La Matrona Hispalense 10: 1-2.

Martínez de Aguirre, B. (1929) La carrera de matrona. Su origen y plan de estudios en la actualidad. La Matrona Hispalense 9: 2-4.

LEGAJO 1180: Padrones benéfico-sanitarios y solicitudes de inscripción al mismo (1910-1936) (Archivo Municipal de San Fernando: A.M.S.).

FIGURA 1.- Portada del libro de Ludwig Piskacek (1929).

FIGURA 2.-Estadística de la matrona Teresa Mateo que representa los partos asistidos de Enero a Septiembre de 1929. Archivo Municipal de San Fernando (Cádiz).

FIGURA 3.- Ilustración del libro de Piskacek (1929). Una matrona explora a una embarazada.

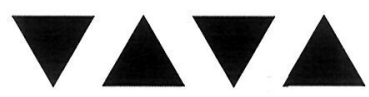

